\title{
Instrumentos de avaliação das complicações na pele periestoma: revisão integrativa
}

Temática: prática baseada em evidência.

Contribuição para a disciplina: 0 enfermeiro tem um papel crucial na avaliação e gestão das complicações do estoma e da pele periestoma. A avaliação precisa da gravidade das complicações na pele periestoma é parte integrante de qualquer decisão diagnóstica e terapêutica. Por isso, o uso de um instrumento que possibilite avaliar, diagnosticar e classificar as condições da pele periestoma de forma padronizada torna-se essencial para a prática clínica.

\section{RESUMO}

Objetivo: identificar e analisar os instrumentos utilizados para a avaliação das complicações da pele periestoma por meio de revisão integrativa. Materiais e métodos: realizou-se revisão nas bases e portais de dados: Biblioteca Virtual da Saúde (BVS), PubMed/ MEDLINE, CINAHL, Scopus e Web of Science, de janeiro a fevereiro de 2018. Durante a busca nas bases de dados, foram utilizados os descritores: estomia, colostomia, dermatite; e as palavras-chave: instrumentos, complicação da pele periestoma e avaliação da pele periestoma. Foram incluídos estudos que atenderam aos seguintes critérios: estar disponível na íntegra, estar publicado em espanhol, inglês e português, sem limite temporal e versar sobre os instrumentos de avaliação das condições da pele periestoma. Resultados: a análise identificou a existência de nove instrumentos, com diferentes parâmetros para descrever a pele periestoma. De maneira geral, estes descrevem as alterações com base na classificação da complicação, na causa dos danos na pele ou nas características clínicas presentes. Os estudos mostraram, ainda, que a maioria dos instrumentos não possui propriedades de medida testadas. Conclusão: dos nove instrumentos de avaliação identificados, apenas quatro possuem propriedades de medidas testadas. 0 enfermeiro deve conhecer os instrumentos confiáveis e válidos disponíveis para descrever e classificar a gravidade dos problemas da pele periestoma, o que contribui para a sistematização de um diagnóstico mais acurado e melhora, portanto, a qualidade do cuidado.

PALAVRAS-CHAVE (FONTE: DECS)

Estomia; colostomia; dermatite; instrumentos; complicação da pele periestoma; avaliação da pele periestoma.

\section{DOI: 10.5294/aqui.2018.18.4.9}

Para citar este artigo / Para citar este artículo / To reference this article

Nunes MLG, Santos VLCG. Instrumentos de avaliação das complicações da pele periestoma: revisão integrativa. Aquichan 2018; 18(2): 477-491. D0I: 10.5294/aqui.2018.18.4.9

$1 \bowtie$ orcid.org/0000-0002-1450-9487. Escola de Enfermagem da Universidade de São Paulo (EEUSP), Brasil. marisgoncalves@usp.br

2 orcid.org/0000-0002-1288-5761. Escola de Enfermagem da Universidade de São Paulo (EEUSP), Brasil. veras@usp.br 


\section{Instrumentos de evaluación de complicaciones de la piel periestoma: revisióón ilintegradora}

\section{RESUMEN}

Objetivo: identificar y analizar las herramientas utilizadas para evaluar las complicaciones de la piel periestoma a través de revisión integradora de la literatura. Materiales y método: la búsqueda se dio en estas bases y portales de datos: Biblioteca Virtual de la Salud (BVS), PubMed/MEDLINE, CINAHL, Scopus y Web of Science, de enero a febrero de 2018. Durante la búsqueda en las bases de datos, se utilizaron los descriptores: ostomía, colostomía, dermatitis y las palabras clave: instrumentos, complicaciones de la piel periestoma y evaluación de la piel periestoma. Se incluyeron estudios que cumplieron los siguientes criterios: que estén disponibles en su totalidad, que estén publicados en español, inglés y portugués, no se tuvo en cuenta el rango de tiempo y tenía que ser acerca de las herramientas de evaluación de las condiciones de la piel periestoma. Resultados: el análisis identificó la existencia de nueve instrumentos con diferentes parámetros para describir la piel periestoma. En general, los instrumentos describen los cambios basados en la clasificación de la complicación, en la causa del daño a la piel o en las características clínicas presentes. Los estudios evidenciaron, además, que la mayoría de los instrumentos no poseen propiedades de medida probadas. Conclusión: de los nueve instrumentos de evaluación identificados, solo cuatro tienen propiedades de medidas probadas. El enfermero debe conocer los instrumentos existentes confiables y válidos para describir y clasificar la gravedad de los problemas de la piel periestoma, lo que aporta a la sistematización de un diagnóstico más preciso y mejora, por lo tanto, la calidad del cuidado.

\section{PALABRAS CLAVE (FUENTE: DeCS)}

Estoma; colostomía; dermatitis; instrumentos; complicaciones de la piel periestoma; evaluación de la piel periestoma. 


\title{
Tools for peristomal skin complications assessment: integrative review
}

\begin{abstract}
Objective: The aim of this study was to identify and analyze the tools used to evaluate the peristomal skin complications by an integrative review. Materials and methods: A literature review was performed through the following databases: Virtual Health Library (BVS), PUBMED/MEDLINE, CINAHL, Scopus and Web of Science, from January to February 2018. During the search in the databases, the following descriptors were used: stoma, colostomy, and dermatitis. The keywords were: tools, peristomal skin complication and peristomal skin assessment. Studies were added to the following inclusion criteria: articles published in Portuguese, English or Spanish, full paper access, no time limit and they had to be about tools for peristomal skin complications assessment. Results: The analysis identified the existence of nine tools with different parameters to describe peristomal skin complications. In general, they describe the peristomal skin changes based on classification, the peristomal skin complications or in the present clinical characteristics. The majority of the tools have not tested psychometric measures. Conclusion: Four out of the nine instruments identified have psychometric measures tested. The nurse should be aware and must know the existing valid and reliable tools to describe and classify how grave is the skin issue. This in order to give an accurate diagnosis and to improve the care quality.
\end{abstract}

KEYWORDS (SOURCE: DeCS)

Ostomy; colostomy; dermatitis; tools; peristomal skin complication; peristomal skin assessment. 


\section{Introdução}

A construção de um estoma intestinal ou urinário continua a ter um papel fundamental no tratamento de doenças como o câncer colorretal e de bexiga, na incontinência anal, trauma, doença inflamatória intestinal, retocolite ulcerativa e nos casos de fistula retovaginal (1-3). As colostomias e as ileostomias são definidas como estomas intestinais de eliminação em que o segmento cólico e ileal são exteriorizados, respectivamente. Já nos estomas urinários, há a exteriorização do conduto urinário, que se justifica para a manutenção da função renal $(3,4)$. Em todas as situações, sem a presença do esfíncter uretral ou anal, o uso de um equipamento coletor faz-se necessário para a coleta do efluente e para a proteção da pele.

0 desenvolvimento de complicações no estoma e na pele periestoma é bastante frequente, e atinge índices acima de 70 \% (5). Estima-se que uma pessoa estomizada experimentará algum tipo de complicação na pele periestoma dentro de dois anos após a cirurgia, apesar dos avanços cirúrgicos e dos cuidados prestados no pós-operatório (6-8). As complicações podem estar associadas a diferentes fatores. Genericamente, tais complicações podem ser minimizadas com o planejamento do local de implantação do estoma, com a técnica cirúrgica correta e adequada, e com a orientação do paciente e da família/cuidadores quanto ao (auto)cuidado (9).

Manter a saúde da pele ao redor do estoma é imprescindível e permanece sendo um desafio para pacientes, cuidadores e equipes de saúde $(10,11)$. A sua importância se dá pelo fato de a pele ser a superfície na qual o equipamento coletor permanecerá aderido (12). A perda da sua integridade reduz a capacidade de aderência da base adesiva, permitindo que o recorrente vazamento do efluente aumente os danos na epiderme e cause desconforto e dor na área afetada, além de aumentar os custos com os cuidados de saúde (12-15). Portanto, a adaptação à nova condição de vida depende substancialmente da preservação da integridade da pele periestoma.

Problemas na pele periestoma são as complicações mais frequentes de quem convive com um estoma (15). Apesar dos avanços em técnicas e produtos para o cuidado, sua incidência e prevalência afetam significativamente a qualidade de vida do estomizado (16-20). 0 tipo mais comum de complicação na pele é a dermatite periestoma associada à umidade, também conhecida como dermatite periestoma, frequentemente resultante da exposição da pele ao efluente do intestinal ou urinário (19-21). Os estomas mais propensos e com maiores coeficientes de danos na pele são as ileostomias, devido principalmente à composição do efluente altamente corrosivo, quando comparadas às colostomias (21).

As causas dos danos na pele periestoma são complexas e multifatoriais, porém uma característica comum é 0 vazamento do efluente entre a barreira protetora do equipamento coletor e a pele. Por sua vez, um conjunto de fatores pode resultar em vazamento do efluente, como o tipo e localização do estoma, o nível de protrusão, o trauma mecânico e a infecção que dificultam o bom ajuste do equipamento coletor, o que leva à fuga do efluente e à irritação da pele (22-24). Os altos índices de danos causados na pele periestoma ressaltam a importância da avaliação precoce que busca identificar os primeiros sinais de lesão, estabelecer 0 diagnóstico e programar o tratamento adequado (25). Ações que são prioritárias para o cuidado do estomizado e que resultam na manutenção de uma pele periestoma saudável $(20,22,24,26)$.

A avaliação da gravidade das condições da pele periestoma é parte integrante de qualquer decisão diagnóstica e apropriada intervenção (27-29). A avaliação clínica e as descrições da etiologia e danos causados na pele periestoma diferem entre os profissionais de saúde, o que dificulta a interpretação e a comunicação entre a equipe. É importante que enfermeiros utilizem uma linguagem comum para descrever as condições da pele periestoma, contribuindo para a sistematização da assistência e para a construção de evidências que fundamentem a prática de cuidados com estomas. Assim, o uso de instrumentos validados para a avaliação da pele periestoma ajudam a padronizar a descrição e a comunicação sobre as suas condições (30-32).

Alguns instrumentos de avaliação das condições da pele periestoma já foram desenvolvidos, mas não há consenso internacional sobre o mais adequado. Diferentemente das ferramentas de avaliação de feridas, aquelas utilizadas para a avaliação da pele periestoma, na grande maioria, não possuem análises de suas propriedades de medida bem como terminologia padronizada, o que torna mais difícil o seu uso e aceitação (33-35). Tendo em vista que os instrumentos existentes apenas começam a ser adaptados e validados em nosso meio, o objetivo deste estudo foi identificar e analisar os instrumentos utilizados para a avaliação das complicações da pele periestoma, por meio de revisão da literatura. 


\section{Materiais e métodos}

Para a elaboração deste estudo, optou-se pelo método de revisão integrativa (RI), que compreende uma ampla abordagem do tema, pois possibilita incluir estudos com diferentes metodologias, como estudos experimentais e não experimentais, estudos empíricos e teóricos (36). Esse método tem um vasto leque de propósitos que se referem a reunir e sintetizar os estudos realizados sobre um determinado tema, a partir dos resultados evidenciados, o que permite gerar uma fonte de conhecimento atual e determina se 0 conhecimento é válido para dar suporte à tomada de decisão e à melhoria da prática clínica $(37,38)$.

Para ser considerada uma pesquisa com rigor metodológico, a RI deve seguir 0 um processo de elaboração de pesquisa, descrito sucintamente em seis fases $(37,39)$ :

- $\quad \mathbf{1}^{\text {a }}$ fase: elaboração da pergunta norteadora para a elaboração da revisão integrativa;

- $\quad 2^{a}$ fase: estabelecimento de critérios para a inclusão e exclusão de estudos/busca de literatura;

- $\quad 3^{a}$ fase: definição das informações a serem extraídas dos estudos selecionados;

- $\quad 4^{a}$ fase: análise crítica dos estudos incluídos;

- $5^{a}$ fase: interpretação dos resultados;

- $\mathbf{6}^{\mathrm{a}}$ fase: apresentação da revisão/síntese do conhecimento.

A definição da pergunta norteadora é a fase mais importante da revisão, pois determina quais serão os estudos incluídos. Nesse sentido, este estudo buscou responder ao seguinte questionamento: quais são os instrumentos para a avaliação das complicações da pele periestoma descritos na literatura?

A busca foi realizada nos meses de janeiro e fevereiro de 2018 nas seguintes bases de dados: Medical Literature Analysis and Retrievel System Online (MEDLINE/PubMed), Cumulative Index to Nursing and Allied Health Literature (CINAHL), Scopus e Web of Science, e nos portais da Biblioteca Virtual da Saúde (BVS). Durante a busca nas bases de dados, os seguintes descritores foram utilizados: "estomia", "colostomia", "dermatite", além das seguintes palavras-chave/MESH: "complicação da pele periestoma", "instrumentos" e "avaliação da pele periestoma". Os descritores foram combinados com operadores boleanos "AND" e "OR" a fim de refinar a busca. Não foi estabelecido limite de tempo cronológico para a busca. Foram incluídos estudos que atenderam aos seguintes critérios: estar disponível na íntegra, estar publicado nos idiomas espanhol, inglês e português, sem limite temporal e versar sobre os instrumentos de avaliação das condições da pele periestoma. Após as exclusões, nove estudos fizeram parte da amostra final.

Para a coleta de dados, as autoras elaboraram um instrumento desenvolvido no programa Microsoft Word. Nele, incluíram-se as seguintes variáveis: autor(es), ano e local de publicação, tipo de estudo e nível de evidência, resumo dos métodos, resumo dos resultados e conclusões.

Com o propósito de avaliar a qualidade metodológica dos estudos incluídos nesta revisão, estes foram submetidos à ferramenta denominada Strengthening the Reporting of Observational Studies in Epidemiology (STROBE), que oferece recomendações sobre como relatar estudos observacionais de forma mais precisa e completa $(40,41)$. A lista de verificação contém 22 itens assim subdivididos: título e resumo, introdução, métodos, resultados, discussão e outras informações. Cada um dos 22 critérios recebe uma pontuação de zero (não atende) a 1 (atende). Após a avaliação dos critérios, cada artigo recebe uma nota de 0 a 22, resultado da somatória dos critérios atendidos (42).

0 instrumento de coleta de dados também continha espaço para a pontuação STROBE. Quanto às evidências dos estudos selecionados, elas foram classificadas em cinco níveis de hierarquia (43):

- nível $\mathbf{1}-1 \mathrm{~A}$ : revisão sistemática de ensaios clínicos controlados; 1B: ensaio clínico controlado randomizado com intervalo de confiança estreito; 1C: resultados terapêuticos do tipo "tudo ou nada";

- nível 2 - 2A: revisão sistemática de estudos de coorte; 2B: estudo de coorte (que inclui ensaio clínico randomizado de menor qualidade); 2C: observação de resultados terapêuticos (outcomes research);

- nível 3 - 3A: revisão sistemática de estudos caso-controle; 3B: estudo caso-controle;

- nível 4 - relato de casos (que incluiu coorte ou caso-controle de menor qualidade);

- nível 5 - opinião de especialistas desprovida de avaliação crítica ou baseada em matérias básicas (estudo fisiológico ou estudo com animais). 
Após a fase de busca nas bases e portais de dados selecionados, os títulos e os resumos foram analisados quanto à concordância com a questão norteadora utilizando os critérios de elegibilidade predefinidos. Definidos os estudos, estes foram acessados na íntegra, e seus dados foram extraídos e analisados por meio de instrumentos de coleta de dados, de avaliação crítica e de nível de evidências, quando pertinente.

\section{Resultados}

Preliminarmente, identificaram-se 267 publicações por meio da busca nas bases de dados e no portal de periódicos selecionados. Após a leitura inicial do título e do resumo, 214 artigos foram eliminados, porque não se encontravam disponíveis na íntegra, foram publicados em duplicidade (em mais de uma base de dados), abordavam outros aspectos da pele periestoma, como definição e descrição das complicações, tratavam de epidemiologia, etiologia, prevenção e tratamento ou não empregavam instrumentos de avaliação ou qualquer forma sistematizada de avaliação das condições da pele periestoma. Quando houve dúvidas quanto à inclusão ou não do artigo, este era lido na íntegra. Ao final do processo de seleção, nove artigos compuseram a revisão, conforme demonstrado na Figura 1.

A análise dos nove artigos da amostra revelou que as publicações se concentraram no período de 1987 a 2014, sendo a PubMed a base com maior número de publicações (oito artigos). A maioria dos artigos é proveniente da Europa (Dinamarca, Inglaterra, Itália, Rússia e Suécia), havendo também representantes da Índia e do Japão. A maior pontuação STROBE foi alcançada pelo estudo prospectivo acerca das propriedades de medida de indicadores de cor, utilizando o domínio Discolouration do instrumento $\mathrm{DET}^{\circledR}$ Score para a validação relacionada ao critério, realizado pela doutora Hiromi Sanada (44) da Universidade de Tóquio. Esse estudo preencheu 17 dos 22 itens do instrumento; trata-se de um estudo prospectivo - nível 2. Embora três artigos não tenham obtido a pontuação STROBE, por serem estudos metodológicos, eles foram mantidos como parte da amostra.

Figura 1. Processo de seleção dos artigos incluídos na revisão integrativa

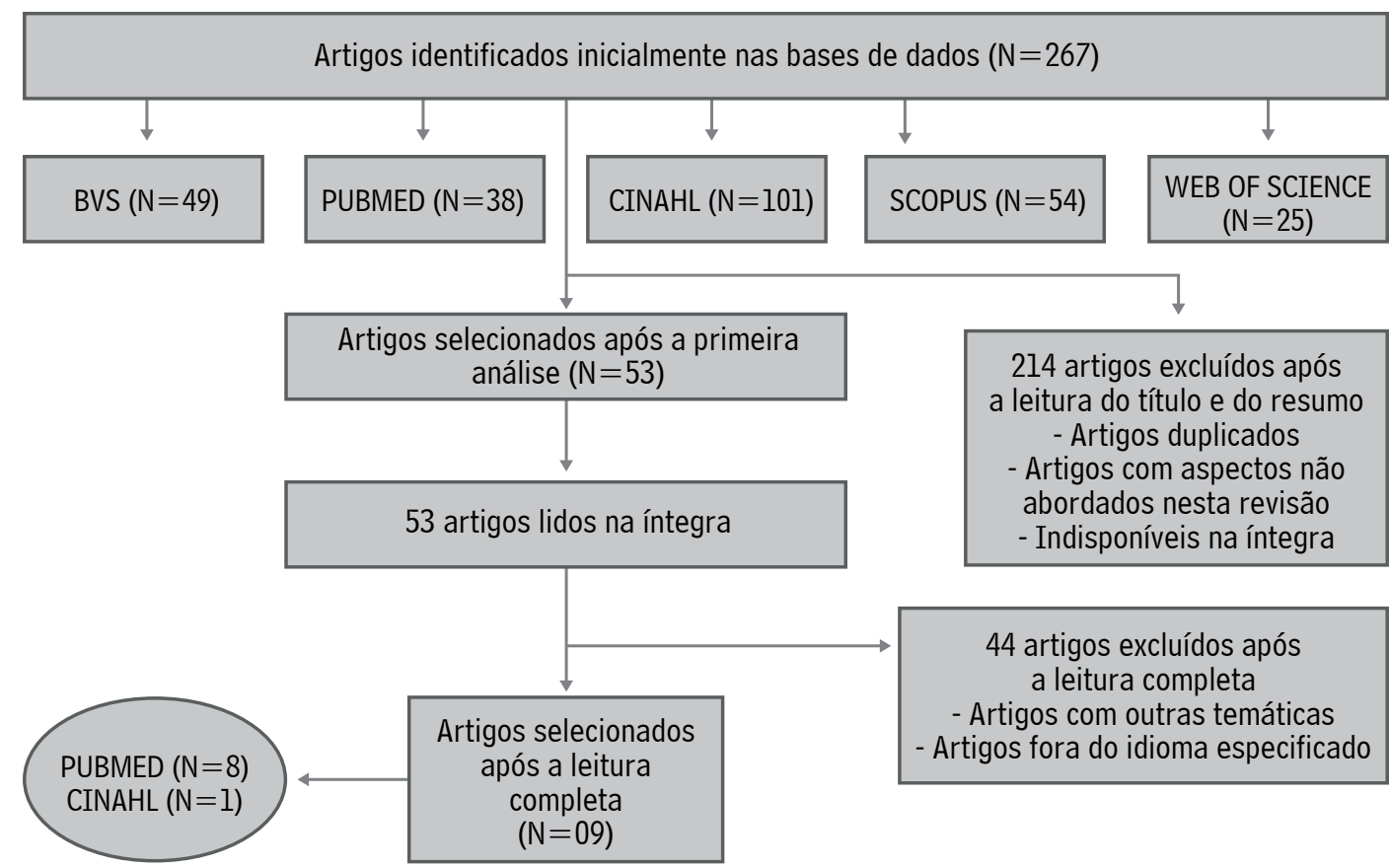

Fonte: elaborado pelas autoras. 
Nos artigos que compuseram a amostra, identificaram-se nove instrumentos desenvolvidos com o propósito de avaliar as complicações da pele periestoma, sendo eles: Classification of Peristomal Skin, Classification of Peristomal Skin (CPS) Changes in Patients with Urostomy, Canadian Ostomy Assessment Guide (CAOG), Study on Peristomal Skin Lesions (SACSTM Instrument), Ostomy Skin Tool, Peristomal Skin Assessment Tool (PSAT), Stoma Care Ostomy Research (SCOR) Index, Color Indicators Using Digi- tal Image Analysis of Peristomal Skin Photographs e o Peristomal Skin Assessment Tool for Patients with Colostomy. Esses instrumentos descrevem as alterações da pele periestoma com base na classificação da complicação (como dermatite por trauma, dermatite por infecção, dermatite alérgica), na causa dos danos na pele (como exposição ao efluente) ou quanto às características clínicas presentes (como coloração vermelha da pele, erosão e ulceração). As características dos nove artigos são apresentadas na Tabela 1.

Tabela 1. Caracterização da produção científica sobre os instrumentos de avaliação na pele periestoma (São Paulo, Brasil, 2018)

\begin{tabular}{|c|c|c|c|c|c|}
\hline $\begin{array}{l}\text { Autor(es), local e } \\
\text { ano de publicação }\end{array}$ & Objetivo & $\begin{array}{l}\text { Tipo de estudo/ } \\
\text { Nível de evidência }\end{array}$ & Métodos & Resultados/Conclusões & $\begin{array}{l}\text { Pontuação } \\
\text { STROBE }\end{array}$ \\
\hline $\begin{array}{l}\text { Herlufsen P et al. } \\
(2006)(10) \\
\text { Dinamarca }\end{array}$ & $\begin{array}{l}\text { Avaliar a frequência, } \\
\text { a diversidade e a } \\
\text { gravidade das condições } \\
\text { da pele periestoma em } \\
\text { estomizados e investigar } \\
\text { como a saúde da pele } \\
\text { era percebida pelos } \\
\text { pacientes. }\end{array}$ & $\begin{array}{l}\text { Estudo transversal/ } \\
\text { Nível de evidência } \\
-2\end{array}$ & $\begin{array}{l}\text { As condições da pele periestoma de } 202 \text { estomizados } \\
\text { foram avaliadas por enfermeiros e dermatologistas } \\
\text { simultaneamente. As alterações da integridade } \\
\text { da pele periestoma foram categorizadas em: leve } \\
\text { (discreta mudança na pele, envolvendo de } 0,1-0,5 \mathrm{~cm} \\
\text { ao redor do estoma); moderada (mudanças na pele, } \\
\text { como ulcerações, em uma área }>2,0 \mathrm{~cm} 2 \text { ) e grave } \\
\text { (comprometimento considerável da pele, o que exige } \\
\text { mudanças do equipamento coletor e cuidado tópico, } \\
\text { tais como uso de medicamentos). }\end{array}$ & $\begin{array}{l}\text { Um total de } 45 \% \text { dos participantes apresen- } \\
\text { tou complicações na pele periestoma, diag- } \\
\text { nosticadas por enfermeiros e dermatologista } \\
\text { ( } 57 \% \text { foram categorizadas como leves; } 33 \% \text {, } \\
\text { moderadas, e } 10 \% \text {, graves). Predominaram } \\
\text { complicações moderadas e graves entre } \\
\text { urostomizados ( } 44 \% \text { e } 22 \% \text {, respectivamen- } \\
\text { te). Dos diagnósticos, } 77 \% \text { estavam relacio- } \\
\text { nados ao contato com o efluente do estoma. } \\
\text { Apenas } 38 \% \text { dos participantes concordaram } \\
\text { que tinham danos na pele e mais de } 80 \% \text { não } \\
\text { procuraram profissionais de saúde. }\end{array}$ & 14 \\
\hline $\begin{array}{l}\text { Borglund E, Nords- } \\
\text { tröm G, Nyman Cr } \\
(1988)(45) \\
\text { Suécia }\end{array}$ & $\begin{array}{l}\text { Avaliar, descrever e } \\
\text { classificar sistemati- } \\
\text { camente as alterações } \\
\text { na pele periestoma em } \\
\text { urostomizados. }\end{array}$ & $\begin{array}{l}\text { Estudo observa- } \\
\text { cional/Nível de } \\
\text { evidência }-2\end{array}$ & $\begin{array}{l}\text { Cinquenta e sete pacientes com conduto ileal foram } \\
\text { avaliados por um estomaterapeuta e um derma- } \\
\text { tologista quanto às características das alterações } \\
\text { macroscópicas da pele periestoma. A documentação } \\
\text { fotográfica das condições da pele compôs a base } \\
\text { para uma classificação sistematizada em três grupos } \\
\text { de alterações: sem sinais de lesões irritativas, lesões } \\
\text { eritematosas-erosivas e lesões pseudoverrucosas. } \\
\text { Posteriormente, as lesões foram classificadas quanto } \\
\text { à gravidade em: ausência de lesão, lesão moderada e } \\
\text { lesão grave. } 0 \text { instrumento não gera uma pontuação } \\
\text { geral. }\end{array}$ & $\begin{array}{l}\text { Quanto aos pacientes examinados de } \\
\text { acordo com o instrumento Classification of } \\
\text { Peristomal Skin (CPS), um terço não apre- } \\
\text { sentou sinais de eritema, erosão ou lesões } \\
\text { pseudoverrucosas, apesar dos achados } \\
\text { comuns como descoloração da pele. Dez dos } \\
13 \text { pacientes com lesões pseudoverrucosas } \\
\text { também exibiram lesões eritematosas-ero- } \\
\text { sivas; lesões eritematoso-erosivas graves } \\
\text { foram reconhecidas em } 7 \% \text { dos pacientes. } \\
\text { O CPS usa definições e termos de acordo com } \\
\text { a terminologia dermatológica, e os autores } \\
\text { esperam que possa ser usado para facilitar a } \\
\text { avaliação e documentação das alterações na } \\
\text { pele periestoma. }\end{array}$ & 08 \\
\hline $\begin{array}{l}\text { Diane St-Cyr (2002) } \\
\text { (46) } \\
\text { Canadá }\end{array}$ & $\begin{array}{l}\text { Avaliar as condições da } \\
\text { pele periestoma e a utili- } \\
\text { dade de um instrumento } \\
\text { de avaliação clínica } \\
\text { entre enfermeiros não } \\
\text { especialistas. }\end{array}$ & $\begin{array}{l}\text { Estudo coorte } \\
\text { prospectivo/Nível } \\
\text { de evidência }-2\end{array}$ & $\begin{array}{l}\text { Durante um período de seis meses, } 18 \text { enfermeiras } \\
\text { avaliaram a utilidade do Canadian Ostomy Asses- } \\
\text { sment Guide (COAG) como ferramenta clínica para } \\
\text { enfermeiros não especialistas. Cinquenta pacientes } \\
\text { foram avaliados três vezes e, em cada visita, o } \\
\text { enfermeiro avaliava o estoma e a pele periestoma. } \\
\text { Em seguida, eram aplicados produtos indicados pelo } \\
\text { instrumento. Cada enfermeiro completou uma ava- } \\
\text { liação final sobre a utilidade do COAG usando uma } \\
\text { escala tipo Likert de } 5 \text { pontos. O COAG possui três } \\
\text { algoritmos: colostomias e ileostomias, urostomias e } \\
\text { problemas comuns da pele periestoma. }\end{array}$ & $\begin{array}{l}\text { Os problemas de pele periestoma foram } \\
\text { observados em } 42 \% \text { da população no } \\
\text { início do estudo (t0), em } 48 \% \text { na visita de } \\
\text { acompanhamento e em } 44 \% \text { na avaliação } \\
\text { final. Entre os enfermeiros participantes, } 78 \\
\% \text { acreditaram que o instrumento COAG foi } \\
\text { útil para a sua prática clínica. }\end{array}$ & 10 \\
\hline
\end{tabular}




\begin{tabular}{|c|c|c|c|c|c|}
\hline $\begin{array}{l}\text { Autor(es), local e } \\
\text { ano de publicação }\end{array}$ & Objetivo & $\begin{array}{l}\text { Tipo de estudo/ } \\
\text { Nível de evidência }\end{array}$ & Métodos & Resultados/Conclusões & $\begin{array}{l}\text { Pontuação } \\
\text { STROBE }\end{array}$ \\
\hline $\begin{array}{l}\text { Bosio G, Pisani F, } \\
\text { Lucibello L et al. } \\
\text { (2007) (33) } \\
\text { Itália }\end{array}$ & $\begin{array}{l}\text { Desenvolver um instru- } \\
\text { mento para classificar } \\
\text { as complicações da pele } \\
\text { periestoma com base na } \\
\text { apresentação clínica e } \\
\text { na localização. }\end{array}$ & $\begin{array}{l}\text { Estudo prospectivo, } \\
\text { observacional/ } \\
\text { Nível de evidência } \\
-2\end{array}$ & $\begin{array}{l}\text { Desenvolvido em oito centros especializados na Itá- } \\
\text { lia, composto por } 339 \text { estomizados com complicações } \\
\text { na pele periestoma, avaliados por estomaterapeutas } \\
\text { e cirurgiões entre } 2003 \text { e } 2006 \text {. As lesões foram } \\
\text { examinadas em intervalos definidos por um período } \\
\text { de dois anos por observações clínicas. }\end{array}$ & $\begin{array}{l}\text { Cinco categorias de lesões (L) foram defini- } \\
\text { das: L1 — lesão com hiperemia; L2 — lesão } \\
\text { erosiva; L3 - lesão ulcerativa; L4 - lesão } \\
\text { fibrinosa/necrótica ulcerativa e LX — lesões } \\
\text { proliferativas (granulomas, depósitos de } \\
\text { oxalato, neoplasia). Os quadrantes ao redor } \\
\text { do estoma foram identificados para facilitar } \\
\text { topografia das lesões. A concordância } \\
\text { interobservadores quanto à classificação } \\
\text { dos diagnósticos foi considerada "excelente" } \\
(K=0,91) \text {. }\end{array}$ & 14 \\
\hline $\begin{array}{l}\text { Jemec GB, Martins } \\
\text { L, Ayello EA, Claes- } \\
\text { sens I, Hansen AS, } \\
\text { Poulsen LH, Sibbald } \\
\text { RG (2011) (30) } \\
\text { Canadá }\end{array}$ & $\begin{array}{l}\text { Avaliar a confiabilidade } \\
\text { inter e intraobservado- } \\
\text { res e o OST — DET®S- } \\
\text { core em comparação } \\
\text { com um grupo "padrão } \\
\text { ouro", definido por um } \\
\text { painel de especialistas. }\end{array}$ & $\begin{array}{l}\text { Estudo metodoló- } \\
\text { gico/Não se aplica } \\
\text { para estudos de } \\
\text { análise psicomé- } \\
\text { trica }\end{array}$ & $\begin{array}{l}\text { Trinta fotografias de complicações da pele } \\
\text { periestoma foram avaliadas por um grupo de vinte } \\
\text { enfermeiros especialistas em dois momentos. Em } \\
\text { ambas as sessões, os enfermeiros atribuíram uma } \\
\text { pontuação utilizando o instrumento DET }{ }^{\circledR} S \text { Score. Um } \\
\text { painel de especialistas composto por cinco membros } \\
\text { avaliou as mesmas } 30 \text { fotografias duas vezes usando } \\
\text { um instrumento de gravidade de } 0 \text { a } 10 \text {. As avalia- } \\
\text { ções foram comparadas para validar as pontuações. } \\
0 \text { DET } @ \text { Score pontua clinicamente as condições da } \\
\text { pele com base em três parâmetros: Descoloração } \\
\text { (D), Erosão/ulceração (E) e Tecido com crescimento } \\
\text { excessivo (T). A pontuação DET } ® \text { varia de } 0 \text { a } 15 \\
\text { pontos de acordo com a soma das pontuações para } \\
\text { cada domínio. }\end{array}$ & 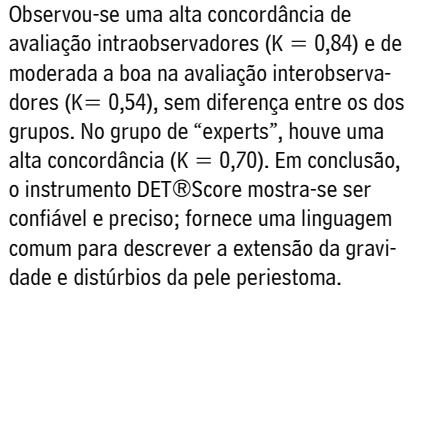 & $\begin{array}{l}\text { Não se } \\
\text { aplica em } \\
\text { estudo de } \\
\text { análise } \\
\text { psicomé- } \\
\text { trica }\end{array}$ \\
\hline $\begin{array}{l}\text { Sodhi JK, Aharma P } \\
\text { (2012) (50) } \\
\text { Índia }\end{array}$ & $\begin{array}{l}\text { Construir um instru- } \\
\text { mento padronizado de } \\
\text { avaliação e prevenção } \\
\text { de complicações da } \\
\text { pele periestoma em } \\
\text { colostomizados. }\end{array}$ & $\begin{array}{l}\text { Estudo metodoló- } \\
\text { gico/Não se aplica } \\
\text { para estudos análi- } \\
\text { se psicométrica }\end{array}$ & $\begin{array}{l}\text { Esse estudo foi desenvolvido conforme as seguintes } \\
\text { fases: fase I — preparação preliminar (revisão de } \\
\text { literatura, seleção de itens que representassem os } \\
\text { parâmetros para a avaliação da pele periestoma); } \\
\text { fase II — validação do primeiro draft pela Técnica } \\
\text { Delphi modificada e alterações de acordo com a } \\
\text { opinião do especialista, e fase III — validação de } \\
\text { conteúdo. Fotos foram adicionadas no instrumento de } \\
\text { acordo com os critérios de classificação. }\end{array}$ & $\begin{array}{l}0 \text { resultado final foi a formulação do Peristo- } \\
\text { mal Skin Assessment Tool (PSAT). Composto } \\
\text { por seis parâmetros de avaliação: cor, tipo } \\
\text { de tecido e tipo de exsudato, sangramento, } \\
\text { margens da ferida e hidratação. } 0 \text { índice de } \\
\text { validade de conteúdo (IVC) do instrumento } \\
\text { PSAT foi de } 0,80 \text {. }\end{array}$ & $\begin{array}{l}\text { Não se } \\
\text { aplica em } \\
\text { estudo de } \\
\text { análise } \\
\text { psicomé- } \\
\text { trica }\end{array}$ \\
\hline $\begin{array}{l}\text { Williams J et al. } \\
(2010)(24) \\
\text { Inglaterra }\end{array}$ & $\begin{array}{l}\text { Identificar os problemas } \\
\text { da pele periestoma } \\
\text { em relação ao uso de } \\
\text { diferentes tipos de } \\
\text { equipamentos coletores } \\
\text { e adjuvantes e comparar } \\
\text { as percepções dos esto- } \\
\text { mizados e enfermeiros } \\
\text { especialistas quanto às } \\
\text { condições de pele. }\end{array}$ & $\begin{array}{l}\text { Estudo descritivo/ } \\
\text { multicêntrico/Nível } \\
\text { de evidência - } 4\end{array}$ & $\begin{array}{l}\text { Usando questionários estruturados, } 80 \text { estomizados } \\
\text { foram entrevistados, e uma fotografia da pele peries- } \\
\text { toma foi tirada; suas respostas foram comparadas } \\
\text { com a avaliação do enfermeiro utilizando o Stoma } \\
\text { Care Ostomy Research (SCOR) Index. }\end{array}$ & $\begin{array}{l}\text { Observou-se que } 68 \% \text { dos pacientes apre- } \\
\text { sentavam problemas na pele, dos quais } 44 \text { \% } \\
\text { eram irritação, } 12 \% \text { ulceração, } 9 \% \text { alergia } \\
\text { e } 3 \% \text { maceração/erosão. Os resultados } \\
\text { demonstraram diferenças significativas entre } \\
\text { a percepção de problemas da pele entre os } \\
\text { estomizados e a observação por enfermeiros } \\
\text { especialistas. Educação do paciente e } \\
\text { acompanhamento regular do enfermeiro } \\
\text { especialista são imperativos. }\end{array}$ & $\begin{array}{c}\text { Não se } \\
\text { aplica em } \\
\text { estudo } \\
\text { descritivo }\end{array}$ \\
\hline $\begin{array}{l}\text { Kalashnikova I, } \\
\text { Achkasov S, Fade- } \\
\text { eva S, Vorobiev G } \\
\text { (2011) (51) }\end{array}$ & $\begin{array}{l}\text { Desenvolver um algo- } \\
\text { ritmo para padronizar } \\
\text { e facilitar a avaliação e } \\
\text { opções de tratamento de } \\
\text { complicações do estoma } \\
\text { e da pele periestoma. }\end{array}$ & $\begin{array}{l}\text { Estudo Prospec- } \\
\text { tivo/Nível de } \\
\text { evidência }-2\end{array}$ & $\begin{array}{l}0 \text { algoritmo foi desenvolvido baseado na literatura } \\
\text { existente, opiniões de profissionais e de uma análise } \\
\text { retrospectiva dos registros de pacientes de um } \\
\text { Centro de Coloproctologia da Russia. } 0 \text { algoritmo } \\
\text { definiu como causas dos danos na pele periestoma: } \\
\text { extravasamento do efluente, infecção da pele, alergia } \\
\text { e doenças preexistentes. A dermatite periestoma foi } \\
\text { avaliada de acordo com as mudanças da coloração } \\
\text { e a gravidade da pele em três grupos: 1) eritema e } \\
\text { maceração; } 2 \text { ) vesículas e erosão; 3) úlceras. Para } \\
\text { testar o seu uso na prática clínica, o algoritmo foi } \\
\text { utilizado por enfermeiras não especialistas para } \\
\text { orientar a avaliação e cuidado em } 1.427 \text { pacientes } \\
\text { ambulatoriais. }\end{array}$ & $\begin{array}{l}\text { Dos } 1.427 \text { pacientes atendidos, } 553 \text { ( } 38,8 \% \text { ) } \\
\text { apresentaram } 742 \text { complicações. Destas, } 355 \\
\text { (47,8 \%) foram classificadas como problemas } \\
\text { na pele periestoma e } 387 \text { ( } 52,2 \% \text { ) como com- } \\
\text { plicações do estoma. Dos } 553 \text { pacientes com } \\
\text { complicações, a mais frequente observada foi } \\
\text { a dermatite periestoma associada à umidade } \\
\text { (184 pacientes, 33,3 \%). Os resultados } \\
\text { desse estudo concluem que os usos dos } \\
\text { algoritmos facilitam a categorização rápida } \\
\text { das complicações e a seleção do tratamento, } \\
\text { embora os autores mencionem que as ava- } \\
\text { liações continuam sujeitas a interpretações e } \\
\text { subjetividades. }\end{array}$ & 14 \\
\hline
\end{tabular}




\begin{tabular}{|c|c|c|c|c|c|}
\hline $\begin{array}{l}\text { Autor(es), local e } \\
\text { ano de publicação }\end{array}$ & Objetivo & $\begin{array}{l}\text { Tipo de estudo/ } \\
\text { Nível de evidência }\end{array}$ & Métodos & Resultados/Conclusões & $\begin{array}{l}\text { Pontuação } \\
\text { STROBE }\end{array}$ \\
\hline $\begin{array}{l}\text { Iizaka S, Asada M, } \\
\text { Koyanagi H, Sasaki } \\
\text { S, Naito A, Konya } \\
\text { C, Sanada H (2014) } \\
\text { (44) } \\
\text { Japão }\end{array}$ & $\begin{array}{l}\text { Avaliar a confiabilidade e } \\
\text { validade dos indicadores } \\
\text { de cor utilizando a aná- } \\
\text { lise de imagens digitais } \\
\text { para a avaliação da pele } \\
\text { periestoma. }\end{array}$ & $\begin{array}{l}\text { Estudo Prospec- } \\
\text { tivo/Nível de } \\
\text { evidência }-2\end{array}$ & $\begin{array}{l}\text { Realizado com } 21 \text { pacientes ( } 14 \text { colostomizados, } 6 \\
\text { ileostomizados e } 1 \text { urostomizado) em quatro ambula- } \\
\text { tórios especializados do Japão. As fotografias foram } \\
\text { processadas utilizando a análise de imagem digital, } \\
\text { que envolveu calibração de cores, processamento } \\
\text { de imagens e cálculo de indicadores. Índice de } \\
\text { eritema (EI), Índice de melanina (MI) e Índice de } \\
\text { hipopigmentação foram criados para representar } \\
\text { graus elevados de coloração vermelha, preta e } \\
\text { branca, respectivamente, e seus valores médios } \\
\text { foram comparados com o domínio Descoloração do } \\
\text { instrumento DET®Score. }\end{array}$ & $\begin{array}{l}\text { O EI e IM da pele periestoma foram signifi- } \\
\text { cativamente maiores que os da pele intacta } \\
\text { ( } n=42 / p<0,001) \text {. Todos os indicadores } \\
\text { de cor em regiões adjacentes e áreas onde } \\
\text { a base foi aplicada foram associados com } \\
\text { o escore de gravidade de descoloração e } \\
\text { escore de intensidade de dor ( } p<0,05) \text {. A } \\
\text { correlação significativa entre a pontuação da } \\
\text { gravidade da descoloração e os indicadores } \\
\text { de cor nesse estudo indicou que a análise de } \\
\text { imagem tinha validade relacionada ao critério } \\
\text { para classificação de cores. Segundo os } \\
\text { autores, os indicadores de cor permitem que } \\
\text { os profissionais executem de forma precisa e } \\
\text { sofisticada a avaliação das condições da cor } \\
\text { da pele periestoma. }\end{array}$ & 17 \\
\hline
\end{tabular}

Fonte: elaborado pelas autoras.

\section{Discussão}

Nove artigos compuseram a amostra desta revisão de literatura, a qual se propôs a identificar os instrumentos de avaliação da pele periestoma. 0 conteúdo dos artigos selecionados refere-se à descrição geral do instrumento utilizado e ao resultado da análise psicométrica testada. Deve-se destacar que alguns instrumentos foram desenvolvidos como primeiras tentativas de sistematizar a avaliação e classificação dos problemas na pele periestoma, e, embora não tenham sido submetidos a testes de validade e confiabilidade, são discutidos neste estudo.

Em 2006, Herlufsen et al. (10) avaliaram a pele periestoma de 202 estomizados com estomas definitivos, na Dinamarca, com 0 objetivo de analisar a frequência, a gravidade e a diversidade de complicações nela presentes. Adicionalmente, os autores pesquisaram como os pacientes percebiam as condições da sua pele comparativamente à avaliação realizada pelos investigadores. Antes de iniciar o estudo, os pesquisadores definiram pela periestoma saudável como a completa ausência de qualquer alteração visível da pele na área periestoma. Quanto à categorização de qualquer alteração de sua integridade, foi realizada de acordo com as características clínicas presentes, em: leve, moderada e grave. Alterações leves eram classificadas como discreta mudança na pele, que envolvia de 0,5 a $1,0 \mathrm{~cm}$ ao redor do estoma, sem necessidade de alterações quanto ao uso do equipamento coletor utilizado; alterações moderadas eram definidas como mudanças na pele, como ulcerações periestoma em uma área superior a 2,0 $\mathrm{cm}^{2}$, necessitando da mudança do equipamento coletor e/ ou indicação de terapia tópica; alterações graves eram aquelas com importante comprometimento da pele, exigindo mudanças do equipamento coletor e cuidado tópico, tais como uso de medicamentos. Essa categorização não gera uma pontuação total, apenas quantifica a frequência média de pacientes com complicações na pele periestoma, segundo a categoria encontrada. Testes de confiabilidade e validade não foram publicados.

Para descrever, definir e classificar os tipos de lesões e sua gravidade, Borglund et al. (45) desenvolveram um sistema de classificação para as complicações da pele periestoma em pacientes com urostomias. A avaliação proposta também foi baseada nas alterações clínicas da pele, distribuídas nos seguintes grupos: sem sinais de lesões irritativas na pele, lesões eritematosas-erosivas e lesões pseudoverrucosas. A proposta incluiu ainda classificação quanto à gravidade: ausência de lesão, lesão moderada e lesão grave, sendo definidas conforme a área: eritematosas menores e maiores que $1 \times 2 \mathrm{~cm}$, respectivamente para moderadas e graves. Um quadro com imagens das diferentes alterações clínicas compõe o instrumento. Embora tenham sido desenvolvidos há 30 anos, testes de confiabilidade e validade também não foram encontrados.

0 estudo (46) que originou o desenvolvimento do Canadian Ostomy Assessment Guide (COAG) teve como principais objetivos: avaliar as mudanças no estado da pele peristoma, o impacto financeiro no custo total dos produtos para cuidados com estomas, a percepção geral do paciente quanto ao bem-estar e à aceitação em relação ao equipamento coletor selecionado e a utilidade do 
COAG como ferramenta clínica para o enfermeiro não especializado. Desenvolvido no Canadá por um grupo de estomaterapeutas, em 1999, o COAG possui três algoritmos de acordo com o tipo de estoma: 1) colostomias e ileostomias, 2) urostomias e 3) problemas comuns da pele periestoma. 0 algoritmo da pele periestoma inclui as seguintes categorias para as complicações: candidíase cutânea, dermatite de contato alérgica, MASD (descrito como dermatite de contato irritativa), maceração, foliculite, psoríase, úlceras por pressão, pioderma gangrenoso, sangramento excessivo e separação mucocutânea. Cada algoritmo contém uma lista de critérios de avaliação com perguntas para confirmar os achados clínicos. Até 0 ano de 2001, 4.500 enfermeiros em todo o Canadá foram capacitados para usar o COAG em sua prática clínica. Porém, tanto os resultados de sua utilização como os de avaliação psicométrica não foram testados.

Desenvolvido por Bosio et al. (33), o instrumento SACS ${ }^{\mathrm{TM}}$ (Studio Alterazioni Cutanee Stomali ou Study on Peristomal Skin Disorders) foi elaborado na Itália, a partir de um estudo prospectivo observacional em oito centros de cuidados com estomas nesse país, desenvolvido entre 2003 e 2006. Os pacientes foram divididos em dois grupos de acordo com o tempo de cirurgia (grupo 1: $<1$ ano; grupo 2: > 1 ano), para diferenciar complicações precoces e tardias. Um total de 656 pacientes estomizados compôs a amostra final, e as lesões na pele periestoma foram examinadas por observações clínicas e imagens digitais em intervalos definidos, ao longo de 24 semanas. Após o período de observação, os membros do grupo de estudo, dos oito centros participantes, validaram a classificação das complicações de pele, durante três conferências de consenso. As classificações foram baseadas em observações e conhecimento sobre o mecanismo das lesões da pele periestoma. Como anteriormente apresentado, o SACS ${ }^{\mathrm{TM}}$ descreve as alterações na pele periestoma de acordo com sua apresentação clínica e localização topográfica em quatro quadrantes ao redor do estoma. As cinco lesões mais comuns observadas no estudo e incluídas no instrumento são: Ll - hiperemia (vermeIhidão da pele periestoma sem perda de substância); L2 — lesão erosiva (com perda de substância que não ultrapassa a derme); L3 - lesão ulcerativa que se estende além da derme; L4 — lesão ulcerativa fibrinosa/lesão necrótica e L5 - lesões proliferativas (granulomas, depósitos de oxalato, neoplasia). Posteriormente à classificação dos diagnósticos do Instrumento SACS ${ }^{\mathrm{TM}}$, foi atestada por um grupo de quatro profissionais que não participaram da construção do instrumento, e a concordância interobservadores foi considerada como quase perfeita ou excelente $(K=0,91)$. Adaptado e validado em 2015, na Turquia (47), a confiabilidade interobservadores foi considerada muito boa ( $K=0,90$; IC $95 \%$ $0,80-0,99)$. Recentemente, o Instrumento SACS ${ }^{\mathrm{TM}}$ foi revisto com o objetivo de classificar lesões que não eram categorizadas pelo instrumento. Uma nova categoria foi incluída: lesão ulcerativa que envolve planos além da fáscia muscular (com ou sem fibrina, necrose, pus ou fístula). A nova versão é denominada SACS 2.0 (48). 0 estudo de adaptação cultural e análise das propriedades psicométricas do SACS ${ }^{\mathrm{TM}}$ se encontra em fase de finalização no Brasil.

Outro esforço para fornecer aos profissionais de saúde um instrumento de classificação das condições da pele periestoma, o Ostomy Skin Tool (OST) (30) foi desenvolvido por um grupo internacional de 12 enfermeiros especialistas em cuidados com estomas, em 2008. A primeira seção do OST pontua clinicamente as condições da pele com base em três parâmetros clínicos: Descoloração (D), Erosão/ulceração (E) e Tecido com crescimento excessivo (T). Esses parâmetros foram identificados como os domínios que descrevem a mais ampla gama de problemas da pele periestoma e são utilizados para calcular a pontuação DET ${ }^{\circledR}$ (resultante da soma das pontuações para cada domínio). Dentro de cada um dos parâmetros, considera-se a extensão da área afetada sob a barreira adesiva, baseada em uma pontuação de 0-3. Quanto à gravidade, cada um dos três domínios é pontuado em uma escala de 1 a 2 . A pontuação total do DET ${ }^{\circledR}$ varia de 0 a 15 pontos. A segunda parte do OST inclui um guia que fornece o diagnóstico da pele periestoma de acordo com a avaliação clínica e as descrições padronizadas. Em 2010, as propriedades de medida do OST foram testadas por Jemec et al. (30). A concordância interobservadores foi considerada moderada $(K=0,54) \mathrm{e}$ excelente para concordância intraobservadores $(K=0,84)$. Recentemente, na Turquia, o OST foi adaptado e validado (49), e foi obtida concordância interobservadores moderada $(K=0,51-0,77)$ excelente para a confiabilidade intraobservadores $(K=0,83-1)$ na avaliação individual dos domínios (área e gravidade). 0 estudo de adaptação cultural e análise das propriedades psicométricas do OST se encontra em fase de finalização no Brasil. 0 estudo de adaptação cultural para a língua portuguesa e a análise das propriedades psicométricas do OST se encontram em fase de finalização no Brasil.

Em 2012, Sodhi e Sharma (50) desenvolveram um estudo metodológico para criar o Peristomal Skin Assessment Tool (PSAT), para a avaliação dos problemas da pele periestoma de colostomizados. A Técnica Delphi modificada foi utilizada para analisar o primeiro draft do instrumento. Após essa fase, um painel de especialistas concebeu a versão final, que consiste em uma lista 
de seis parâmetros baseados, como anteriormente, na avaliação clínica da pele: coloração, sangramento, margens da lesão, tipo de tecido e tipo de exsudato e hidratação, com critérios de pontuação máxima até 18. Fotos foram adicionadas ao instrumento de acordo com os critérios de classificação. 0 índice de validade de conteúdo (IVC) do PSAT foi de 0,8. Demais testes de confiabilidade e validade não foram realizados.

Em um estudo descritivo e multicêntrico, conduzido por William et al. (24) com o objetivo de identificar e classificar as complicações da pele periestoma relacionadas ao uso de equipamentos coletores e adjuvantes, 80 estomizados foram avaliados por meio do Stoma Care Ostomy Research (SCOR) Index. Esse instrumento combina dois parâmetros: a avaliação da aparência clínica da pele e o diagnóstico da complicação. Os autores definiram pele periestoma saudável como a completa ausência de qualquer diferença visível entre a pele periestoma e a pele abdominal oposta. Os problemas da pele periestoma foram classificados e codificados quanto à gravidade, de leve a grave. Quanto às complicações, estas foram categorizadas como alergia, ulceração, eritema, maceração/erosão e irritação. 0 instrumento SCOR não apresenta sistema de pontuação. Análises psicométricas do instrumento ainda não foram publicadas.

A fim de desenvolver uma abordagem uniforme para o diagnóstico e tratamento de complicações do estoma e da pele, e que fosse de fácil uso por enfermeiros não especialistas, Kalashnikova et al. (51) desenvolveram um algoritmo para diagnóstico e escolha do tratamento em complicações em estomas, em 2004. Os distúrbios na pele periestoma foram diagnosticados com base em observações clínicas que utilizam dois parâmetros: mudanças na cor da pele periestoma e gravidade do dano causado na pele. Os autores consideraram ainda como essas condições afetam a seleção do tratamento. De acordo com a gravidade das lesões, a dermatite periestoma foi dividida em três grupos: 1) eritema, maceração, 2) vesículas, erosão e 3) úlceras. Estudos de análises psicométricas do algoritmo precisam ser conduzidos.

A cor é considerada um indicador importante na avaliação de danos da pele periestoma e sua gravidade, bem como na sua restauração. Diante disso, Sanada et al. (44) desenvolveram um estudo prospectivo para avaliar a confiabilidade e validade dos indicadores de cores que usam fotografias digitais da pele periestoma. Conforme as observações clínicas da pele, Índice de eritema (EI), Índice de melanina (MI) e Índice de hipopigmentação foram criados para representar graus aumentados de colora- ções vermelha, preta e branca, respectivamente, e seus valores médios foram calculados em relação aos valores da pele intacta. Os índices de eritema e de melanina da pele periestoma foram significativamente maiores que os da pele intacta ( $<<0,001)$. Esse método objetivo e simples teve reprodutibilidade adequada e validade relacionada ao critério, e pode ser útil para a avaliação da pele periestoma. Todos os indicadores de cor na região da base adesiva foram associados à gravidade do escore do domínio Descoloração do instrumento $\mathrm{DET}^{\circledR}$ Score. Os autores acreditam que esse método de avaliação ajudará na tomada de decisões clínica, no monitoramento e na melhoria do efeito do cuidado prestado, bem como na habilidade de avaliação de enfermeiros especializados na prática diária.

Comparando os instrumentos apresentados nesta revisão integrativa, podemos verificar que os autores utilizaram diferentes parâmetros de avaliação e uma variedade de nomenclaturas para classificar as complicações da pele periestoma, o que pode dificultar a escolha, pelo enfermeiro, de qual instrumento é o mais apropriado para usar na prática clínica. Adicionalmente, há falta de estudos de análises psicométricas ou poucas propriedades de medida testadas nos instrumentos de avaliação aqui apresentados, o que pode justificar que, embora eles tenham sido desenvolvidos e introduzidos ao longo dos anos, nenhum deles obteve uso clínico difundido ou foi considerado universalmente aceito (33-35).

Como limitação deste estudo, pode-se mencionar a não obtenção de uma amostra de estudos com alto rigor metodológico e baixo nível de evidência; portanto, faz-se necessário o desenvolvimento de estudos mais robustos sobre a temática.

\section{Conclusão}

Em suma, este estudo possibilitou identificar nove instrumentos internacionais, que foram desenvolvidos com a proposta de classificar as complicações da pele periestoma. Destes, apenas quatro instrumentos possuem propriedades de medidas testadas, sendo que o OST e o SACS ${ }^{\mathrm{TM}}$ possuem mais estudos de adaptação cultural e validação para uso em outras línguas.

É essencial que os profissionais de saúde avaliem sistematicamente, registrem e comuniquem as condições da pele periestoma, de forma consistente e padronizada. Vários instrumentos de avaliação já foram desenvolvidos, mas não há consenso internacional sobre 0 mais adequado. Os instrumentos descritos neste estu- 
do utilizam diferentes maneiras para descrever a gravidade das complicações da pele periestoma, o que dificulta a padronização de terminologia e a comparação de resultados.

A utilização de um instrumento validado para monitorar os danos na pele periestoma contribui para a sistematização da as- sistência ao possibilitar uma linguagem padronizada entre os profissionais. Sua utilização consiste em uma poderosa ferramenta de comunicação, clara e consistente, e viabiliza a continuidade do cuidado e a melhor monitorização dos resultados alcançados.

Conflito de interesse: nenhum declarado.

\section{Referências}

1. Habr-Gama A, Neto AS, Araújo SEA. Estomas intestinais: aspectos conceituais e técnicos. Em: Santos VLCG, Cesaretti IUR, organizadoras. Assistência em Estomaterapia: Cuidando de pessoas com estomias. $2^{\mathrm{a}}$ ed. São Paulo: Editora Atheneu; 2015. p. 41-46.

2. Paula PR, Speranzini MB. Colostomias e ileostomias. Em: Paula MAB, Paula PR, Cesaretti IUR, organizadoras. Estomaterapia em foco e o cuidado especializado. São Caetano do Sul. SP: Yendis Editora; 2014. p. 15-32.

3. Rodrigues P. Estomas urinários: aspectos conceituais e técnicos. Em: Santos VLCG, Cesaretti IUR, organizadoras. Assistência em Estomaterapia: Cuidando de pessoas com estomias. 2a ed. São Paulo: Editora Atheneu; 2015. p. 47-61.

4. Sharon F. Care of patients with urinary stomas. Em: Breckman B, organizadora. Stoma Care and Rehabilitation. Londres: Churchill Livingstone; 2005. p. 93-104.

5. Boyles A, Hunt S. Care and management of a stoma: maintaining peristomal skin health. Br J Nurs. 2016 (Stoma Supplement);25(17):S14-21. DOI: 10.12968/bjon.2016.25.17.S14

6. Beitz JM, Colwell JC. Management approaches to stomal and peristomal complications. a narrative descriptive study. J Wound Ostomy Continence Nurs. 2016;43(3):263-8. DOI: 10.1097/WON.0000000000000215

7. Kwiatt M, Kawata M. Avoidance and management of stomal complications. Clin Colon Rectal Surg. 2013;26(2):112-21. DOI: $10.1055 / \mathrm{s}-0033-1348050$

8. Almutairi D, LeBlanc K, Alavi A. Peristomal skin complications: what dermatologists need to know. International Journal of Dermatology. 2017;57(3):257-64. DOI: 10.1111/ijd.13710

9. Zulkowski K, Ayello EA, Stelton S. Practice implications for peristomal skin assessment and care from the 2014 World Council of Enterostomal Therapists International Ostomy Guideline. Adv Skin Wound Care. 2015;28(6):275-84. DOI: 10.1097/01.ASW.0000465374.42350.0f

10. Herlufsen P, Olsen AG, Carlsen B, Nybaek H, Karlsmark T, Laursen TN et al. Study of peristomal skin disorders in patients with permanent stomas. Br J Nurs. 2006;15(16):854-62. DOI: 10.12968/bjon.2006.15.16.21848

11. Blessy J, Kim MY, Daniel K. Risk factors associated with peristomal skin complications: integrative literature. World Council of Enterostomal Therapists Journal. 2018 [citado em 26 jun. 2018];38(2):41-2. Disponível em: http:// web.b.ebscohost.com/ehost/pdfviewer/pdfviewer?vid=2\&sid=67415270-f952-4263-bbe4-c4fdc9e17013\%40pdc-vsessmgr03

12. Rolstad BS, Erwin-Toth PL. Peristomal skin complications: prevention and management. Ostomy Wound Manage. 2004 [citado em 12 maio 2018];50(9):68-77. Disponível em: https://www.o-wm.com/content/peristomal-skin-complicationsprevention-and-management

13. Steinhagen E, Colwell J, Cannon LM. Intestinal stomas - postoperative stoma care and peristomal skin complications. Clin Colon Rectal Surg. 2017;30:184-92. DOI: 10.1055/s-0037-1598159

14. English E, Claessens I. How peristomal skin disorders impact on ostomy care. World Council of Enterostomal Therapists Journal. 2008;28(2)(Suppl.):3-7. 
15. Taneja C, Netsch D, Rolstad BS, Inglese G, Lamerato L, Oster G et al. Clinical and economic burden of peristomal skin complications in patients with recent ostomies. J Wound Ostomy Continence Nurs. 2017;44(4):1-8. DOI: 10.1097/ WON.0000000000000339

16. Colwell JC, Pittman J, Raizman R, Salvadalena G. A randomized controlled trial determining variances in ostomy skin conditions and the economic impact (ADVOCATE Trial). J Wound Ostomy Continence Nurs. 2018;45(1):37-42. DOI: 10.1097/WON.0000000000000389

17. Nybaek H, Jemec GBE. Skin problems in stoma patients. J Eur Acad Dermatol Venereol. 2010;24:249-57. DOI: $10.1111 /$ j.1468-3083.2010.03566.x

18. Maydick-Youngberg D. A descriptive study to explore the effect of peristomal skin complications on quality of life of adults with permanent ostomy. Ostomy Wound Management. 2017 [citado em 25 maio 2018];63(5):10-23. Disponível em: https://www.o-wm.com/article/descriptive-study-explore-effect-peristomal-skin-complications-quality-lifeadults-permanent

19. Colwell JC, McNichol L, Boarini J. North America Wound, Ostomy, and Continence and Enterostomal Therapy Nurses Current Ostomy Care Practice Related to Peristomal Skin Issues. J Wound Ostomy Continence Nurs. 2017;44(3):257-61. DOI: 10.1097/WON.0000000000000324

20. Meisner S, Lehur PA, Moran B, Martins L, Jemec GB. Peristomal skin complications are common, expensive, and difficult to manage: a population-based cost modeling study. PLoS One. 2012;7(5):1-9. DOI: 10.1371/journal.pone.0037813

21. Persson E, Berndtsson I, Carlsson E, Hallén AM, Lindholm E. Stoma related complications and stoma size - a 2-year follow up. Colorectal Dis. 2010;12(10):971-6. DOI: 10.1111/j.1463-1318.2009.01941.x

22. Doctor K, Colibaseanu TD. Peristomal skin complications: causes, effects, and treatments. Chronic Wound Care Management and Research. 2017;4:1-6. DOI: 10.2147/CWCMR.S93615

23. Salvadalena GD. The Incidence of stoma and peristomal complications during the first 3 months after ostomy creation. J Wound Ostomy Continence Nurs. 2013;40(4):400-6. DOI: 10.1097/WON.0b013e318295a12b

24. Williams J, Gwillam B, Sutherland N, Matten J, Hemmingway J, Ilsey H, et al. Evaluating skin care problems in people with stomas. Br J Nurs. 2010;19(17)Suppl:S6-15. DOI: 10.12968/bjon.2010.19.Sup7.78569

25. Wound, Ostomy and Continence Nurses Society. Peristomal skin complications: Clinical resource guide. 2016 [citado em 12 maio 2018]:1-43. Disponível em: https://c.ymcdn.com/sites/www.wocn.org/resource/resmgr/Publications/Peristomal_Skin_Complication.pdf

26. Salvadalena G. Peristomal skin conditions. Em: Carmel JE, Colwell JC, Goldberg MT, editores. WOCN Core Curriculum: Ostomy Management. Philadelphia, PA: Wolters Kluwer; 2016. p. 176-90.

27. Beitz JM, Colwell JC. Stomal and peristomal complications: Prioritizing management approaches in adults. J Wound Ostomy Continence Nurs. 2014;41(5):445-54. DOI: 10.1097/WON.0000000000000052

28. Stelton S, Zulkowski K, Ayello EA. Practice implications for peristomal skin assessment and care from the 2014 World Council of Enterostomal Therapists International Ostomy Guideline. Adv Skin Wound Care. 2015;28(6):275-84. DOI: 10.1097/01.ASW.0000465374.42350.0f

29. Colwell JC, Beitz J. Survey of Wound, Ostomy and Continence (WOC) nurse clinicians on stomal and peristomal complications. J Wound Ostomy Continence Nurs. 2007;34(1):57-69. DOI: 10.1097/00152192-200701000-00010

30. Jemec GB, Martins L, Claessens I, Ayello EA, Hansen AS, Poulsen LH, et al. Assessing peristomal skin changes in ostomy patients. Validation of the Ostomy Skin Tool. Br J Dermatol. 2011;164(2):330-5. DOI: 10.1111/j.1365-2133.2010.10093.x

31. Perrin A. Using the Ostomy Skin Tool to assist communication between ostomy care nurses. World Council of Enterostomal Therapists Journal. 2008;28(2)-(Suppl.):14-5.

32. WCET International Ostomy Guideline Recommendations. World Council of Enterostomal Therapists Journal. 2014 [citado em 23 mar. 2018];34(2):26-8. Disponível em: https://www.wcetn.org/assets/Publications/wcet_april-june_2014f\%20 iog\%20recommandations.pdf 
33. Bosio G, Pisani F, Lucibello L Fonti A, Scrocca A, Morandell C, et al. A proposal for classifying peristomal skin disorders: results of a multicenter observational study. Ostomy Wound Manage. 2007 [citado em 10 maio 2018];53(9):38-43. Disponível em: https://www.o-wm.com/content/a-proposal-classifying-peristomal-skin-disorders-results-a-multicenterobservational-study

34. Haugen V, Ratliff CR. Tools for assessing peristomal skin complications. J Wound Ostomy Continence Nurs. 2013;40(2):131-4. DOI: 10.1097/WON.0b013e31828001a7

35. Gray M, Colwell JC, Doughty D, Goldberg M, Hoeflok J, Manson A, et al. Peristomal moisture-associated skin damage in adults with fecal ostomies. A comprehensive review and consensus. J Wound Ostomy Continence Nurs. 2013;40(4):38999. DOI: 10.1097/WON.0b013e3182944340

36. Soares CB, Hoga LAK, Peduzzi M, Sangaleti C, Yonekura T, Silva DRAD. Revisão integrativa: conceitos e métodos utilizados na enfermagem. Rev Esc Enferm USP. 2014;48(2):335-45. DOI: 10.1590/S0080-6234201400002000020

37. Crossetti MGO. Revisão integrativa de pesquisa na enfermagem o rigor científico que lhe é exigido. [editorial]. Rev Gaúcha Enferm. 2012;33(2):8-9. Disponível em: https://lume.ufrgs.br/handle/10183/94920

38. Ercole FF, Melo LS, Alcoforado CLGC. Integrative Review versus Systematic Review. REME. Rev Min Enferm. 2014 [citado em 30 maio 2018];18(1):12-4. DOI: 10.5935/1415-2762.20140001

39. Souza LMM, Marques-Vieira CMA, Severino SSP, Antunes AV. Metodologia de revisão integrativa da literatura em enfermagem. Revista em investigação em enfermagem. 2017 [citado em 28 jun. 2018];ser. II(21):17-26. Disponível em: http://hdl.handle.net/20.500.12253/1311

40. Galvão TF, Silva MT, Garcia LP. Ferramentas para melhorar a qualidade e a transparência dos relatos de pesquisa em saúde: guias de redação científica. Epidemiol Serv Saude. 2016;25(2):427-36. DOI: 10.5123/S1679-49742016000200022

41. Malta M, Cardoso LO, Bastos FI, Magnanini MMF, Silva CMFP. Iniciativa STROBE: subsídios para a comunicação de estudos observacionais. Rev Saúde Pública. 2010;44(3):559-65. DOI: 10.1590/S0034-89102010000300021

42. Elm E von, Altman DG, Egger M, Pocock SJ, Gøtzsche PC, Vandenbroucke JP et al. Strengthening the reporting of observational studies in epidemiology (STROBE) Statement: Guidelines for reporting observational studies. Int J Surg. 2014;4(10):1495-9. DOI: 10.1371/journal.pmed.0040296

43. Centre for Evidence Based Medicine. Oxford Centre for Evidence-based Medicine -Level of Evidence. 2009 [citado em 1 jun. 2018]. Disponível em: http://www.cebm.net/oxford-centre-evidence-based-medicine-levels-evidence-march-2009

44. Sanada H. The reliability and validity of color indicators using digital Image analysis of peristomal skin photographs: results of a preliminary prospective clinical study. Ostomy Wound Manage. 2014 [citado em 21 abril 2018];60(30):1229. Disponível em: https://www.o-wm.com/article/reliability-and-validity-color-indicators-using-digital-image-analysis-peristomal-skin-photo

45. Borglund E, Nordstrm G, Nyman CR. Classification of peristomal skin changes in patients with urostomy. J Am Acad Dermatol. 1988;19:623-8. DOI: 10.1016/S0190-9622(88)70215-7

46. St-Cyr D. An evaluation of Canadian Ostomy Assessment Guide. Ostomy Wound Manage. 2002 [citado em 6 jun. 2018];48(8):1-6. Disponível em: https://www.o-wm.com/content/an-evaluation-canadian-ostomy-assessment-guide

47. Ay A, Bulut H. Assessing the validity and reliability of the peristomal skin lesion assessment instrument adapted for use in Turkey. Ostomy Wound Manage. 2015 [citado em 6 jun. 2018];61(8)26-34. Disponível em: https://www.o-wm. com/article/assessing-validity-and-reliability-peristomal-skin-lesion-assessment-instrument-adapted-use

48. Antonini M, Militello G, Manfredda S, Arena R, Veraldi S, GasperiniS. A revised version of the original SACS Scale for peristomal skin disorders classification. World Council of Enterostomal Therapists Journal. 2016 [citado em 10 jun. 2018].;36(3):22-9. Disponível em: http://web.b.ebscohost.com/ehost/detail/detail?vid=14\&sid=67415270-f952-4263bbe4-c4fdc9e17013\%40pdc-v-sessmgr03\&bdata=JnNpdGU9ZWhvc3QtbGl2ZSZzY29wZT1 zaXRl\#AN=118464919\&db=ccm 
49. Harputlu D, Özsoy AS. Turkish reliability and validity of the Ostomy Skin Tool. J Nur Healthcare. 2017 [citado em 13 jun. 2018].;2(4):1-5. Disponível em: https://www.opastonline.com/wp-content/uploads/2017/10/turkish-reliabilityand-validity-of-the-ostomy-skin-tool-jnh-17.pdf

50. Sodhi JK, Sharma P. A Methodological study to develop a peristomal skin assessment tool for patients with colostomy in selected Hospital, Ludhiana, Punjab. Int J Nurs Educ. 2012 [citado em 20 jun. 2018];4(2):209-12. Disponível em: http://web.b.ebscohost.com/ehost/detail/detail?vid=17\&sid=67415270-f952-4263-bbe4-c4fdc9e17013\%40pdc-v-sess mgr03\&bdata=JnNpdGU9ZWhvc3QtbGl2ZSZzY29wZT1zaXRl\#db=ccm\&AN=104394696

51. Kalashnikova I, Achkasov S, Fadeeva S, Vorobiev G. The Development and use of algorithms for diagnosing and choosing treatment of ostomy complications: results of a prospective evaluation. Ostomy Wound Manage. 2011 [citado em 20 jun. 2018];57(1):20-7. Disponível em: https://www.o-wm.com/content/development-and-use-algorithms-diagnosing-and-choosing-treatment-ostomy-complications-result 